\title{
The clinical features of Ehlers-Danlos syndrome type VII due to a deletion of 24 amino acids from the proa1(I) chain of type I procollagen
}

\author{
W G COLE*, R EVANS + , AND D O SILLENCE
}

From * the Department of Paediatrics, University of Melbourne, Australia; $\dagger$ the Department of Paediatrics $\mathbb{\otimes}$ Memorial Hospital, Hastings, New Zealand; and $\ddagger$ the Department of Public Health Biology, University of Sydney, Australia.

SUMMARY The clinical features and progress of a child with the type VII form of EhlersDanlos syndrome due to a deletion in the proa1(I) of type I procollagen were studied. The child was born with bilateral dislocations of hips and knees and all other joints were markedly hypermobile. Persistent severe joint instability was the major clinical abnormality. She had a depressed nasal bridge with prominent paranasal folds and deeply set eyes with mild hypertelorism and micrognathia. The skin was soft, moderately hyperelastic, and sagged over the face and knees. Skin fragility and easy bruising appeared when she started walking. Electron microscopy of the dermis showed irregular collagen fibrils.

Ehlers-Danlos syndrome (EDS) is a heterogeneous genetic disorder of the connective tissues. ${ }^{1}$ The major phenotypic features include joint hypermobility and skin changes such as thinness, hyperextensibility, and fragility. In humans, the type VII form of this syndrome (EDS VII) is characterised by severe joint laxity with multiple dislocations and subluxations. ${ }^{2}$ This form of EDS has been shown to involve impaired removal of the amino terminal $\mathrm{N}$ propeptides from procollagen..$^{2-4}$

In the EDS VIIA variant, the $\mathrm{N}$-propeptides are incompletely removed because of reduced activity of procollagen $\mathrm{N}$-proteinase. ${ }^{2}$ Partially processed proal(I) and proa2(I) chains of type I procollagen are found in the tissues of such patients. These biochemical findings also exist in dermatosparaxis ('torn skin'), a recessively inherited disease of cattle, sheep, and cats.'

The biochemical defects in the EDS VIIB variant involve structural alterations of the proal(I) or proo2(I) chains, which prevent cleavage of the abnormal chain by procollagen $\mathrm{N}$-proteinase. ${ }^{1} \mathrm{~A}$ peptide deletion, including the $\mathrm{N}$-proteinase cleavage site, has been reported in the proa2(I) chain of a

Received for publication 27 August 1986. Accepted for publication 22 September 1986. child with EDS VIIB ${ }^{3}$ and a probable structura岕 defect in the same region of this chain has also bee reported in another child. ${ }^{4}$

We previously reported the biochemical feature $\$$ of a child with EDS VIIB. ${ }^{5}$ The child was shown to be heterozygous for a deletion of 24 amino acidos (residues 136 to 159) from the prodl(I) chain. Theo deleted peptide corresponded to the sequence nor은 mally coded by exon 46 of the proal(I) gene. The peptide deletion included the N-proteinase cleavage site and partially processed proal(I) chains were present in the dermis. In this paper we describe thes clinical features, progress, and collagen morphology of this child.

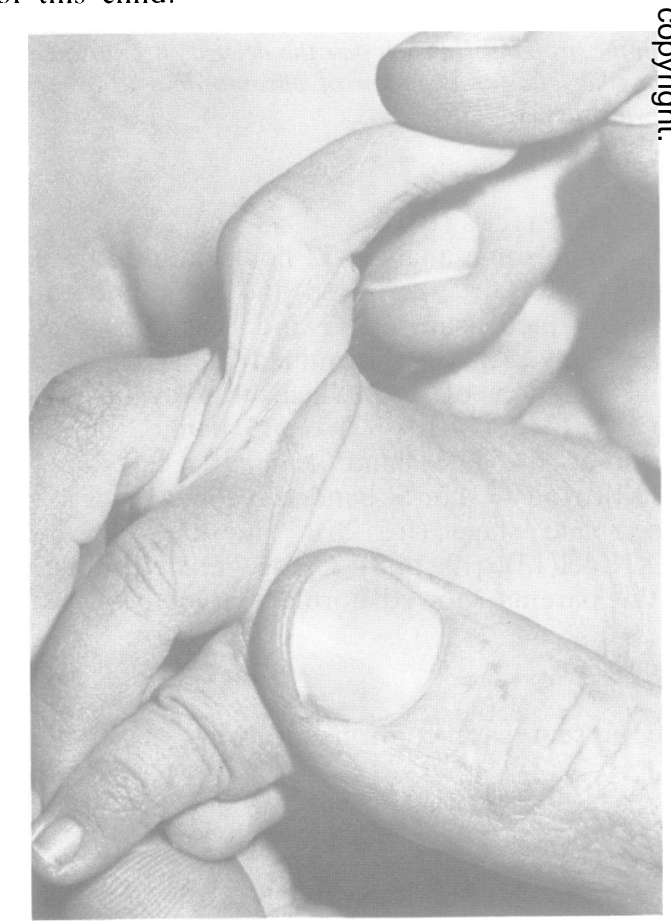

FIG 1 Hand appearance at six months. All joints were lax. The interphalangeal joints could be hyperextended due to laxity of the volar plates and other anterior structures of the fingers. 


\section{Case report}

CIINICAL HISTORY

The proband was the first child of a healthy and unrelated 32 year old father and 22 year old mother. The pregnancy was normal and the membranes did not rupture prematurely. She was born by normal vaginal delivery at 40 weeks' gestation. Apgar scores were 8 at one minute and 9 at five minutes. The birth weight was $3.97 \mathrm{~kg}$ (75th centile), length $53 \mathrm{~cm}$ ( 75 th centile), and head circumference $37 \mathrm{~cm}$ (98th centile).

She was in good condition at birth. She was noted to have bilateral cephalhaematomata. There was a large loose skin fold at the nape of the neck and her skin was soft and moderately hyperelastic. Both hips were dislocated and could be easily reduced, but the reductions could not be maintained in a splint. The knees were also dislocated with the tibiae displaced forwards. They were easily reduced when the knees were flexed but redislocated when extended. All other joints were lax and most could be subluxed (fig 1). The spine was of normal shape. She was generally hypotonic. Ophthalmological examinations revealed no abnormalities.

\section{PROGRESS}

She showed normal fine motor, mental, and social development, but markedly delayed gross motor development. For example, at the age of two years she had the motor development of a six month old child. The delayed motor development appeared to
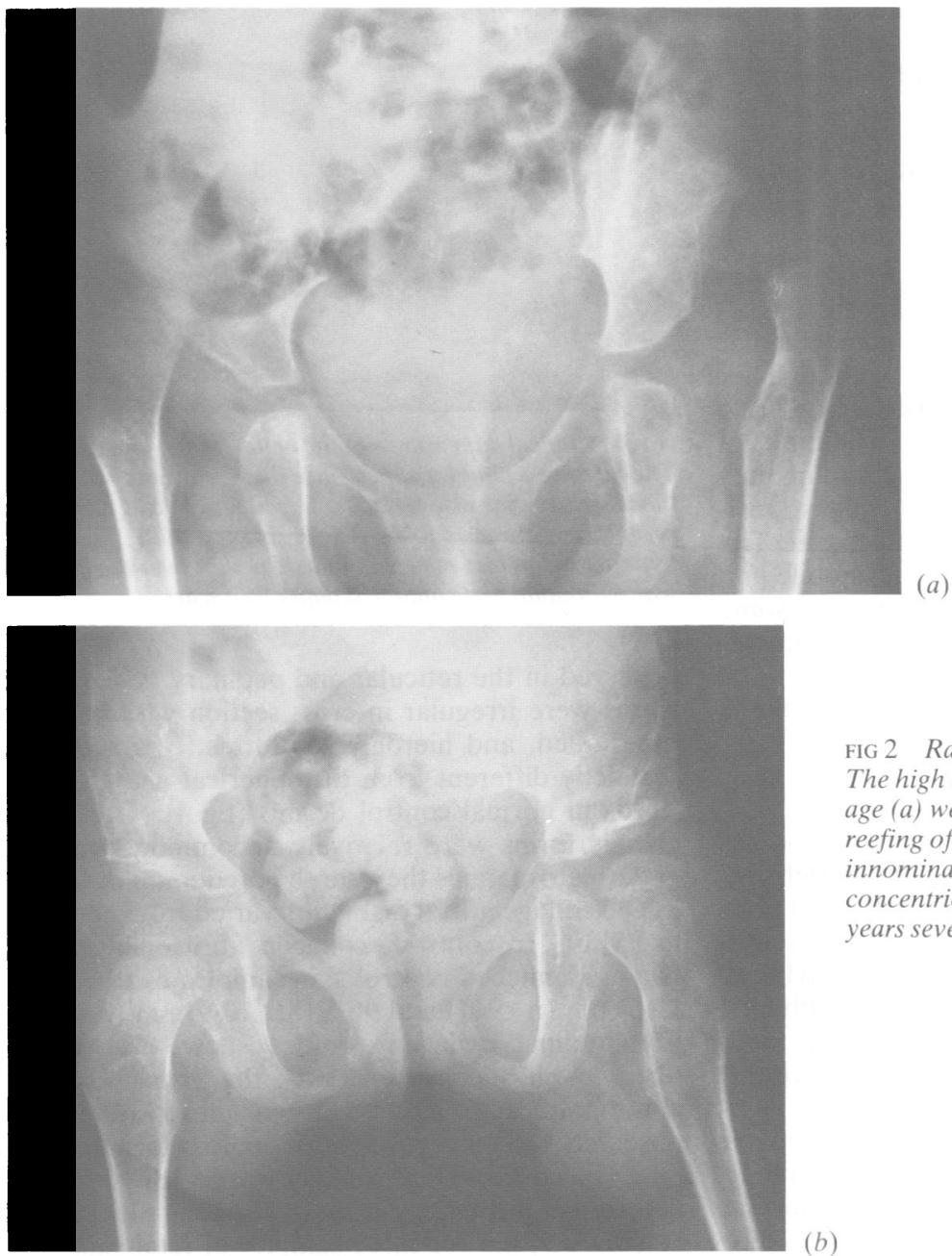

FIG 2 Radiographical appearance of the hips. The high dislocations of the hips at four years of age (a) were reduced and stabilised by bilateral reefing of the redundant joint capsules and innominate osteotomies. The femoral heads are concentric with good acetabular coverage at four years seven months of age (b). 


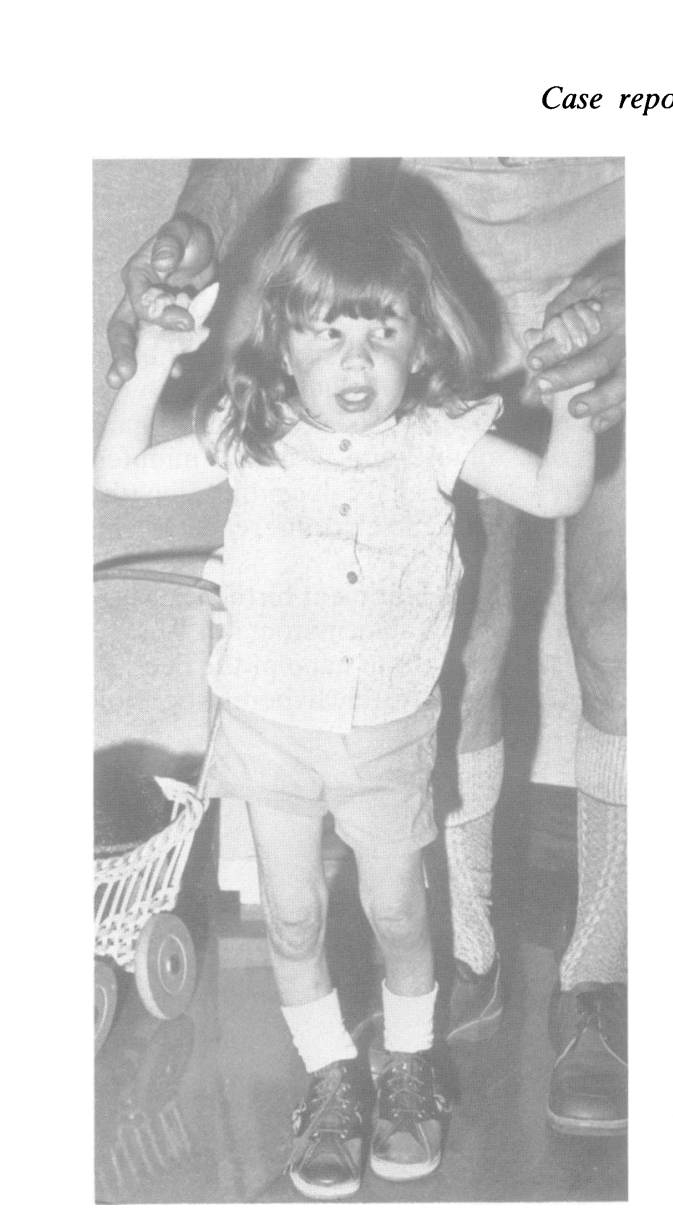

FIG 3 Clinical appearance of the child at four years of age. The face has a chubby appearance due to the laxity of the facial tissues. She also has a depressed nasal bridge, prominent paranasal folds, deeply set eyes, mild hypertelorism, and micrognathia. The skin over the knees was redundant and contained paper thin scars.

observed in the reticular and papillary dermis. The fibrils were irregular in cross section with bilobed $\frac{3}{2}$ multilobed, and hieroglyphic forms, ${ }^{78}$ which were. distinctly different from the spherical and elliptica? fibrils in normal control dermis (fig 4). The hieroglyphic forms were relatively uncommon, whereas in dermatosparaxis they are the predominant form. $\frac{\text {. }}{2}$

The collagen fibril diameters varied from about $35^{5}$. to $95 \mathrm{~nm}$. In contrast, collagen fibril diameters inf two age matched control skin samples were 88 (SD $11.6) \mathrm{nm}(\mathrm{n}=947)$ and $96.5($ SD 10.9) $\mathrm{nm}(\mathrm{n}=981)$ Longitudinal collagen profiles showed indefinitew fibril margins consistent with the cross sectiona $F$ abnormalities. Faint collagen striations were observed with a regular D periodicity of about 5 T $\mathrm{nm}$. The control D periodicity was 55 (SD 2.6) $\mathrm{nm}$ ? determined using 26 samples of postnatal skin, ando $58($ SD $0 \cdot 9) \mathrm{nm}$ for three samples of fetal skin. morphology. Abnormal collagen fibrils were

HISTOLOGICAL FEATURES phosphate buffer containing $2 \cdot 5 \%$ glutaraldehyde. resin. ${ }^{6}$ Ultra-thin sections, stained with uranyl ace tate and lead citrate, were examined using a Phillips EM400 transmission electron microscope. Specimen height and hysteresis corrections were made for the purposes of morphometry.

The epidermal-dermal junction appeared to be normal. Dermal fibroblasts were present in

be directly related to hypotonia and unstable or dislocated joints. An attempt to reduce the hips, using skin traction followed by a plaster hip spica, was unsuccessful. The hips were easily reduced but gross joint instability (fig 2a). At the age of four years she had bilateral innominate osteotomies with reefing of the redundant capsule of the hip joints. Stable reductions were achieved and the hips were immobilised for six weeks in a hip spica followed by

PRESENT STATUS

She was re-examined in June 1986 at the age of four
years seven months and shown to have the following clinical features. She was small (height $98 \mathrm{~cm}, 3 \mathrm{rd}$ centile; weight $13.2 \mathrm{~kg},<3 \mathrm{rd}$ centile). Her short stature was due, at least in part, to a progressive right thoracolumbar scoliosis. She had a depressed nasal bridge with prominent paranasal folds and deeply (fig 3 ). Her skin was hyperelastic with sagging of the skin over her face and knees. She had paper thin scars over some of her bony prominences and a history of easy bruising. A large inguinal hernia was She was able to walk independently. Her hips were stable and radiographs showed that the femor-
al heads were concentrically reduced and acetabular development was satisfactory (fig $2 b$ ). Although her knees were still grossly unstable she was able to
stand and walk without knee splintage. The joints of collapsed into severe plano-abducto-valgus positions. These foot deformities impaired her gait. The unstable. The shoulder joints, left elbow, and some of the interphalangeal joints were dislocatable.

The parents have had a second child who is clinically normal.

A thin sliver of skin was fixed in $0.1 \mathrm{~mol} / \mathrm{l}$ sodium Small pieces of the specimen were processed with 


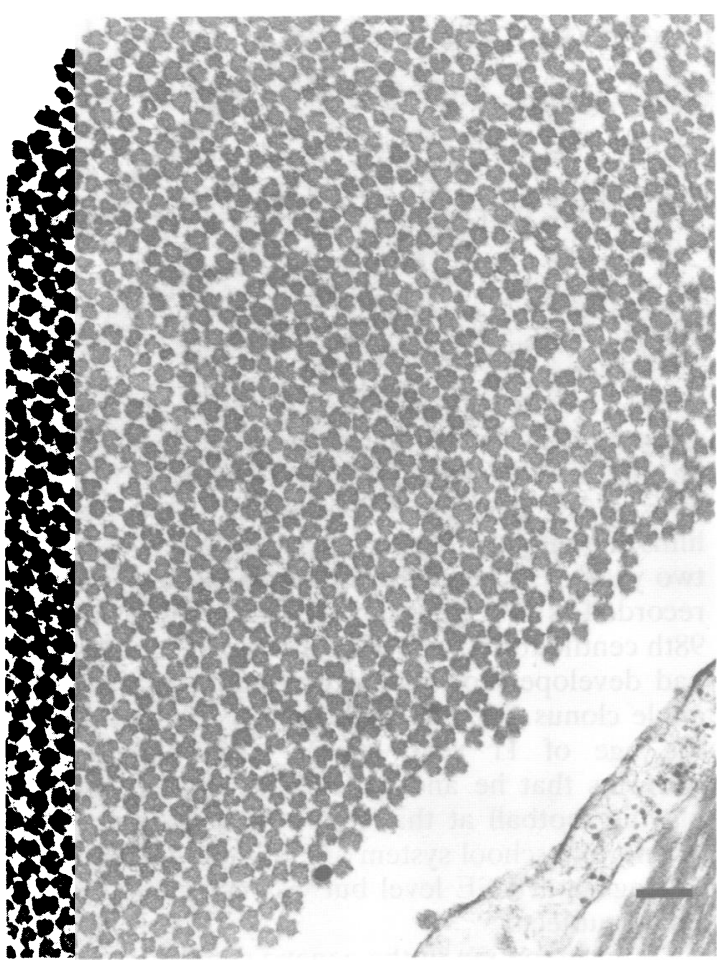

FIG 4 Transverse section of collagen fibrils of the skin. The fibrils are irregular in outline and vary widely in diameter. Horizontal bar $=100 \mathrm{~nm}$. (Original magnification $\times 21500$.)

\section{Discussion}

The clinical phenotype of our child with EDS VII was similar to the reported phenotypes of other children with this form of EDS. ${ }^{2-49}$ In all cases the principle feature was gross joint instability and multiple dislocations which were obvious at birth. The present study indicates that joint instability persists, at least for the first five years of life. Stable reductions of the dislocated hips were achieved by operative procedures after unsuccessful attempts using splints and plasters. The dislocated knees improved spontaneously but were still grossly unstable. Progressive deformities of the feet were an increasing problem and the thoracolumbar scoliosis is likely to progress.

The histological abnormalities of the dermal collagen in our child were similar to those described by Eyre $\mathrm{et}^{3}$ in their child with a peptide deletion in the proa2(I) chain. In both cases the collagen fibrils resembled a loosely wound rope. It is likely that the persistence of $\mathrm{pN}$-collagen (procollagen lacking the carboxy terminal C-propeptide) was responsible for the disruption of the normal fibril structure. Loss of a cross linking site in the deleted peptide may also have contributed to this abnormality. ${ }^{5}$ In contrast. Steinmann $\mathrm{et}^{\mathrm{t}} \mathrm{l}^{\mathrm{t}}$ reported that the dermal collagen fibrils were relatively normal in shape and size in their nine year old child with a probable structural defect in the proc2(I) chain, but some irregularity of the collagen fibres had been observed at an earlier age. ${ }^{7}$

Overall, these patients appear to be sufficiently similar to suggest that a correlation exists between the persistence of one or more of the $\mathrm{N}$-propeptides and the EDS VII phenotype. Despite this apparent correlation, we do not understand why the human disease produces major manifestations in joints, whereas the skin is mainly affected in animals with dermatosparaxis.

We wish to thank Mr G Lamb for providing us with details of the orthopaedic care and for collecting the biopsies.

\section{References}

' Prockop DJ. Kivirikko KI. Heritable diseases of collagen. $N$ Engl J Med 1984:311:376-86.

- Lichtenstein JR. Martin (jR. Kohn LD. Byers PH McKusick VA. Defect in conversion of procollagen to collagen in a form of Ehlers-Danlos syndrome. Science 1973:182:298-300.

Eyre DR. Shapiro FD. Aldridge JF. A heterozygous collagen defect in a variant of the Ehlers-Danlos syndrome type VII. $J$ Biol (hem 1985:260:11322-9.

+ Steinmann B. Tuderman L. Peltonen L. Martin GR. McKusick VA. Prockop DJ. Evidence of a structural mutation of procollagen type $I$ in a patient with the Ehlers-Danlos syndrome type Vil. J Biol (hem 1980):255:8887-93.

Cole WG. Chan D. Chambers GW. Walker ID. Bateman JF Deletion of 24 amino acids from the pro- $\alpha$ l(I) chain of type $I$ procollagen in a patient with the Ehlers-Danlos syndrome type VII. J Biol Chem 1986:261:5496-5013.

- Sillence DO. Bone dssplasias: genetic and ultrastructural studies with special reference to osteogenesis imperfecta. Ann Arbor: University Microfilms-International. 1980.

Williams B. Cranley S. Doty S. Lichtenstein J. Morphological observations on connective tissue from individuals with procollagen peptidase deficiency (Ehlers-Danlos type VII syndrome). Am J Hum Genet 1973:25:86A

* Holbrook KA. Byers PH. Structural abnormalities in the dermal collagen and elastic matrix from the skin of patients with inherited connective tissue disorders. J Invest Dermatol 1982:79:7-16S.

"Lichtenstein JR. Kohn LD. Martin GR. et al. Procollagen peptidase deficiency in a form of the Ehlers-Danlos syndrome. Trans Assoc Am Phwsicians 1973:88:333-9.

Correspondence and requests for reprints to $\mathrm{Dr}$ W G Cole, Department of Paediatrics, University of Melbourne, Royal Children's Hospital, Flemington Road, Parkville, Victoria 3052, Australia. 